\title{
A NEW FRAMEWORK TO APPROACH VIZING'S CONJECTURE
}

\author{
Boštjan Brešar ${ }^{a, b}$, Bert L. Hartnell ${ }^{c}$, Michael A. Henning $^{d}$ \\ Kirsti Kuenzel $^{e}$ and Douglas F. Rall ${ }^{f}$ \\ ${ }^{a}$ Faculty of Natural Sciences and Mathematics \\ University of Maribor, Slovenia \\ ${ }^{b}$ Institute of Mathematics, Physics and Mechanics \\ Ljubljana, Slovenia \\ ${ }^{c}$ Saint Mary's University, Halifax, Nova Scotia, Canada \\ ${ }^{d}$ Department of Mathematics and Applied Mathematics \\ University of Johannesburg, Johannesburg, South Africa \\ ${ }^{e}$ Department of Mathematics, Western New England University \\ Springfield, MA, USA \\ ${ }^{f}$ Department of Mathematics, Furman University, Greenville, SC, USA
}

\begin{abstract}
We introduce a new setting for dealing with the problem of the domination number of the Cartesian product of graphs related to Vizing's conjecture. The new framework unifies two different approaches to the conjecture. The most common approach restricts one of the factors of the product to some class of graphs and proves the inequality of the conjecture then holds when the other factor is any graph. The other approach utilizes the so-called Clark-Suen partition for proving a weaker inequality that holds for all pairs of graphs. We demonstrate the strength of our framework by improving the bound of Clark and Suen as follows: $\gamma(X \square Y) \geq$ $\max \left\{\frac{1}{2} \gamma(X) \gamma_{t}(Y), \frac{1}{2} \gamma_{t}(X) \gamma(Y)\right\}$, where $\gamma$ stands for the domination number, $\gamma_{t}$ is the total domination number, and $X \square Y$ is the Cartesian product of graphs $X$ and $Y$.
\end{abstract}

Keywords: Cartesian product, total domination, Vizing's conjecture, Clark and Suen bound.

2010 Mathematics Subject Classification: 05C69, 05C76.

\section{REFERENCES}

[1] R. Aharoni and T. Szabó, Vizing's conjecture for chordal graphs, Discrete Math. 309 (2009) 1766-1768. doi:10.1016/j.disc.2008.02.025 
[2] A.M. Barcalkin and L.F. German, The external stability number of the Cartesian product of graphs, Bul. Akad. Stiinte RSS Moldoven. 94 (1979) 5-8.

[3] B. Brešar, Improving the Clark-Suen bound on the domination number of the Cartesian product of graphs, Discrete Math. 340 (2017) 2398-2401. doi:10.1016/j.disc.2017.05.007

[4] B. Brešar, Vizing's conjecture for graphs with domination number 3 - a new proof, Electron. J. Combin. 22(3) (2015) \#P3.38.

[5] B. Brešar, P. Dorbec, W. Goddard, B.L. Hartnell, M.A. Henning, S. Klavžar and D.F. Rall, Vizing's conjecture: a survey and recent results, J. Graph Theory 69 (2012) $46-76$. doi:10.1002/jgt.20565

[6] B. Brešar and D.F. Rall, Fair reception and Vizing's conjecture, J. Graph Theory 61 (2009) 45-54. doi:10.1002/jgt.20366

[7] W.E. Clark and S. Suen, An inequality related to Vizing's conjecture, Electron. J. Combin. 7 (2000) \#N4.

[8] P. Erdős and A. Rényi, On the evolution of random graphs, Publ. Math. Inst. Hungar. Acad. Sci. 5 (1960) 17-61.

[9] B.L. Hartnell and D.F. Rall, Vizing's conjecture and the one-half argument, Discuss. Math. Graph Theory 15 (1995) 205-216. doi:10.7151/dmgt.1018

[10] J.W. Moon and L. Moser, Almost all $(0,1)$ matrices are primitive, Studia Sci. Math. Hungar. 1 (1966) 153-156.

[11] M. Pilipczuk, M. Pilipczuk and R. Škrekovski, Some results on Vizing's conjecture and related problems, Discrete Appl. Math. 160 (2012) 2484-2490. doi:10.1016/j.dam.2012.06.011

[12] S. Suen and J. Tarr, An improved inequality related to Vizing's conjecture, Electron. J. Combin. 19(1) (2012) \#P8.

[13] L. Sun, A result on Vizing's conjecture, Discrete Math. 275 (2004) 363-366. doi:10.1016/j.disc.2003.09.003

[14] V.G. Vizing, Some unsolved problems in graph theory, Russian Math. Surveys 23(6) (1968) 125-141. doi:10.1070/RM1968v023n06ABEH001252

[15] S. Zerbib, An improved bound in Vizing's Conjecture, Graphs Combin. 35 (2019) $1401-1404$. doi:/10.1007/s00373-019-02083-6

Received 21 September 2019

Revised 5 December 2019 Accepted 15 December 2019 